\title{
Complete monotonicity, completely monotonic degree, integral representations, and an inequality related to the exponential, trigamma, and modified Bessel functions
}

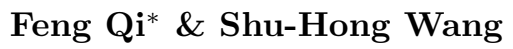 \\ College of Mathematics, Inner Mongolia University for Nationalities, \\ Tongliao City, Inner Mongolia Autonomous Region, 028043, China \\ *Corresponding author E-mail: qifeng618@gmail.com,qifeng618@hotmail.com, qifeng618@qq.com \\ *Corresponding author URL: http: // qifeng618. wordpress. com
}

Copyright (C2014 Qi and Wang. This is an open access article distributed under the Creative Commons Attribution License Creative Commons Attribution License, which permits unrestricted use, distribution, and reproduction in any medium, provided the original work is properly cited.

\begin{abstract}
In the paper, the authors verify the complete monotonicity of the difference $e^{1 / t}-\psi^{\prime}(t)$ on $(0, \infty)$, compute the completely monotonic degree and establish integral representations of the remainder of the Laurent series expansion of $e^{1 / z}$, and derive an inequality which gives a lower bound for the first order modified Bessel function of the first kind. These results show us some new properties and relations of the exponential, trigamma, the first kind modified Bessel functions and the hypergeometric series.
\end{abstract}

Keywords: property; connection; completely monotonic function; completely monotonic degree; integral representation; difference; exponential function; trigamma function; hypergeometric series; inequality; modified Bessel function

\section{Introduction}

In [3, Lemma 2], the inequality

$\psi^{\prime}(t)<e^{1 / t}-1$

on $(0, \infty)$ was obtained and applied, where $\psi(t)$ stands for the digamma function which may be defined by the logarithmic derivative

$\psi(t)=[\ln \Gamma(t)]^{\prime}=\frac{\Gamma^{\prime}(t)}{\Gamma(t)}$ 
and $\Gamma(t)$ is the classical Euler gamma function which may be defined for $\Re z>0$ by

$\Gamma(z)=\int_{0}^{\infty} t^{z-1} e^{-t} \mathrm{~d} t$

The derivatives $\psi^{\prime}(z)$ and $\psi^{\prime \prime}(z)$ of $\psi(z)$ are respectively called the tri- and tetra-gamma functions. As a whole, the derivatives $\psi^{(k)}(z)$ for $k \in\{0\} \cup \mathbb{N}$ are called the polygamma functions.

The first aim of this paper is to generalize the inequality (1.1) to complete monotonicity of a difference between both sides of (1.1).

Our first result can be formulated as Theorem 1.1 below.

Theorem 1.1. The function

$h(t)=e^{1 / t}-\psi^{\prime}(t)$

is completely monotonic, that is, $(-1)^{k-1} h^{(k-1)}(t) \geq 0$ for $k \in \mathbb{N}$, on $(0, \infty)$ and

$\lim _{t \rightarrow \infty} h(t)=1$

The notion "completely monotonic degree" was innovated in [2, Definition 1] which may be regarded as a slight but essential modification of [5, Definition 1.5]. This may be further fixed as follows.

Definition 1.1. Let $f(x)$ be a completely monotonic function on $(0, \infty)$ and denote $f(\infty)=\lim _{x \rightarrow \infty} f(x)$. If for some $r \in \mathbb{R}$ the function $x^{r}[f(x)-f(\infty)]$ is completely monotonic on $(0, \infty)$ but $x^{r+\varepsilon}[f(x)-f(\infty)]$ is not for any positive number $\varepsilon>0$, then we say that the number $r$ is the completely monotonic degree of $f(x)$ with respect to $x \in(0, \infty)$; if for all $r \in \mathbb{R}$ each and every $x^{r}[f(x)-f(\infty)]$ is completely monotonic on $(0, \infty)$, then we say that the completely monotonic degree of $f(x)$ with respect to $x \in(0, \infty)$ is $\infty$.

For convenience, the notation $\operatorname{deg}_{\mathrm{cm}}^{x}[f(x)]$ was designed in [2, p. 9890] to denote the completely monotonic degree $r$ of $f(x)$ with respect to $x \in(0, \infty)$.

It is clear that the completely monotonic degree $\operatorname{deg}_{\mathrm{cm}}^{x}[f(x)]$ of any completely monotonic function $f(x)$ on $(0, \infty)$ is at leat 0 . It may be proved that the completely monotonic degree $\operatorname{deg}_{\mathrm{cm}}^{x}[f(x)]$ equals $\infty$ if and only if $f(x)$ is nonnegative and identically constant.

The second aim of this paper is to compute the completely monotonic degree and to establish integral representations of the remainder of the Laurent series expansion of the exponential function $e^{1 / z}$.

Our second result may be stated as the following theorem.

Theorem 1.2. For $k \in\{0\} \cup \mathbb{N}$ and $z \neq 0$, let

$H_{k}(z)=e^{1 / z}-\sum_{m=0}^{k} \frac{1}{m !} \frac{1}{z^{m}}$.

1. The completely monotonic degree of $H_{k}(t)$ on $(0, \infty)$ meets

$\operatorname{deg}_{\mathrm{cm}}^{t}\left[H_{k}(t)\right]=k+1$.

2. For $\Re z>0$, the function $H_{k}(z)$ has the integral representations

$H_{k}(z)=\frac{1}{k !(k+1) !} \int_{0}^{\infty}{ }_{1} F_{2}(1 ; k+1, k+2 ; t) t^{k} e^{-z t} \mathrm{~d} t$

and

$H_{k}(z)=\frac{1}{z^{k+1}}\left[\frac{1}{(k+1) !}+\int_{0}^{\infty} \frac{I_{k+2}(2 \sqrt{t})}{t^{(k+2) / 2}} e^{-z t} \mathrm{~d} t\right]$,

where the hypergeometric series

${ }_{p} F_{q}\left(a_{1}, \ldots, a_{p} ; b_{1}, \ldots, b_{q} ; x\right)=\sum_{n=0}^{\infty} \frac{\left(a_{1}\right)_{n} \cdots\left(a_{p}\right)_{n}}{\left(b_{1}\right)_{n} \cdots\left(b_{q}\right)_{n}} \frac{x^{n}}{n !}$ 
for $b_{i} \notin\{0,-1,-2, \ldots\}$, the shifted factorial $(a)_{0}=1$ and

$(a)_{n}=a(a+1) \cdots(a+n-1)$

for $n>0$ and any real or complex number $a$, and the modified Bessel function of the first kind

$I_{\nu}(z)=\sum_{k=0}^{\infty} \frac{1}{k ! \Gamma(\nu+k+1)}\left(\frac{z}{2}\right)^{2 k+\nu}$

for $\nu \in \mathbb{R}$ and $z \in \mathbb{C}$.

As an application of Theorems 1.1 and 1.2, the following inequality for the first order modified Bessel function of the first kind $I_{1}$ may be derived.

Theorem 1.3. For $t>0$, we have

$I_{1}(t)>\frac{(t / 2)^{3}}{1-e^{-(t / 2)^{2}}}$

\section{Proofs of Theorem 1.1}

We supply two proofs of Theorem 1.1.

First proof. From the well known formula

$\psi^{(n)}(z)=(-1)^{n+1} \int_{0}^{\infty} \frac{u^{n}}{1-e^{-u}} e^{-z u} \mathrm{~d} u$

for $\Re z>0$ and $n \in \mathbb{N}$, see [1, p. 260, 6.4.1], it is ready that $\lim _{t \rightarrow \infty} \psi^{(n)}(t)=0$ for $n \in \mathbb{N}$. So, the limit $(1.3)$ may be deduced immediately and, by

$\left(e^{1 / t}\right)^{(i)}=(-1)^{i} e^{1 / t} \frac{1}{t^{2 i}} \sum_{k=0}^{i-1} a_{i, k} t^{k}$

for $i \in \mathbb{N}$ and $t \neq 0$, where $a_{i, k}=\left(\begin{array}{c}i \\ k\end{array}\right)\left(\begin{array}{c}i-1 \\ k\end{array}\right) k$ ! for $0 \leq k \leq i-1$, in [13, Theorem 2.1],

$h^{(i)}(t)=\left(e^{1 / t}\right)^{(i)}-\psi^{(i+1)}(t)=(-1)^{i} e^{1 / t} \sum_{k=0}^{i-1} \frac{a_{i, k}}{t^{2 i-k}}-\psi^{(i+1)}(t) \rightarrow 0$

for $i \in \mathbb{N}$ as $t \rightarrow \infty$.

Utilizing the recurrence formula

$\psi^{(n)}(z+1)=\psi^{(n)}(z)+(-1)^{n} \frac{n !}{z^{n+1}}$

in $[1$, p. 260, 6.4.7] and calculating reveal

$$
\begin{aligned}
h(t+1)-h(t) & =e^{1 /(t+1)}-e^{1 / t}+\psi^{\prime}(t)-\psi^{\prime}(t+1) \\
& =e^{1 /(t+1)}-e^{1 / t}+\frac{1}{t^{2}} \\
& =\frac{1}{t^{2}}+\sum_{k=0}^{\infty} \frac{1}{(k+1) !}\left[\frac{1}{(t+1)^{k+1}}-\frac{1}{t^{k+1}}\right] \\
{[h(t+1)-h(t)]^{(i)} } & =(-1)^{i} \frac{(i+1) !}{t^{i+2}}+\sum_{k=0}^{\infty} \frac{(-1)^{i}(i+k) !}{(k+1) ! k !}\left[\frac{1}{(t+1)^{i+k+1}}-\frac{1}{t^{i+k+1}}\right],
\end{aligned}
$$

and

$(-1)^{i}[h(t+1)-h(t)]^{(i)}=\frac{(i+1) !}{t^{i+2}}+\sum_{k=0}^{\infty} \frac{(i+k) !}{(k+1) ! k !}\left[\frac{1}{(t+1)^{i+k+1}}-\frac{1}{t^{i+k+1}}\right]$ 


$$
\begin{aligned}
& <\frac{(i+1) !}{t^{i+2}}+\sum_{k=0}^{2} \frac{(i+k) !}{(k+1) ! k !}\left[\frac{1}{(t+1)^{i+k+1}}-\frac{1}{t^{i+k+1}}\right] \\
& =\frac{i !}{12 t^{i+3}(t+1)^{i+3}} f_{i}(t),
\end{aligned}
$$

where

$$
\begin{aligned}
f_{i}(t)= & 6(i+1) t(t+1)\left[(t+1)^{i+2}+t^{i+2}\right]-12 t^{2}(t+1)^{2}\left[(t+1)^{i+1}-t^{i+1}\right]-(i+1)(i+2)\left[(t+1)^{i+3}-t^{i+3}\right] \\
= & 6(i+1) t(t+1)\left[\begin{array}{c}
i+2 \\
\ell=0
\end{array}\left(\begin{array}{c}
i+2 \\
\ell
\end{array}\right) t^{\ell}+t^{i+2}\right]-12 t^{2}(t+1)^{2} \sum_{\ell=0}^{i}\left(\begin{array}{c}
i+1 \\
\ell
\end{array}\right) t^{\ell}-(i+1)(i+2) \sum_{\ell=0}^{i+3}\left(\begin{array}{c}
i+3 \\
\ell
\end{array}\right) t^{\ell} \\
= & \frac{(i-1)(i+4)(i+5)}{2}\left[\frac{(2-i)(i+3)}{3} t-i\right] t^{2}-i(i+1)(i+5) t \\
& -\sum_{\ell=4}^{i}\left[(i+1)(i+2)\left(\begin{array}{c}
i+3 \\
\ell
\end{array}\right)-6(i+1)\left(\begin{array}{c}
i+3 \\
\ell-1
\end{array}\right)+12\left(\begin{array}{c}
i+3 \\
\ell-2
\end{array}\right)\right] t^{\ell} \\
= & \frac{(i-1)(i+4)(i+5)}{2}\left[\frac{(2-i)(i+3)}{3} t-i\right] t^{2}-i(i+1)(i+5) t \\
& -(1+i)(2+i)-(i+4)(i+5) \sum_{\ell=4}^{i} \frac{(i-\ell+1)(i-\ell+2)}{\ell(i-\ell+5)}\left(\begin{array}{c}
i+3 \\
\ell-1
\end{array}\right) t^{\ell}
\end{aligned}
$$

and an empty sum is understood to be nil. As a result, the function $f_{i}(t)$ is negative and

$$
(-1)^{i}[h(t+1)-h(t)]^{(i)}=(-1)^{i}[h(t+1)]^{(i)}-(-1)^{i}[h(t)]^{(i)}<0
$$

for all $i \geq 0$ and $t \in(0, \infty)$. Hence, by consecutive recursion and (2.3),

$$
(-1)^{i}[h(t)]^{(i)} \geq(-1)^{i}[h(t+1)]^{(i)} \geq(-1)^{i}[h(t+2)]^{(i)} \geq \cdots \geq(-1)^{i}[h(t+k)]^{(i)} \geq(-1)^{i} \lim _{k \rightarrow \infty}[h(t+k)]^{(i)}=0
$$

for $i \in \mathbb{N}$ and $t \in(0, \infty)$. This implies that the function $h(t)$ is decreasing on $(0, \infty)$. Combining this monotonicity with (1.3) gives $h(t)>1$ on $(0, \infty)$. In conclusion, by definition, the function $h(t)$ is completely monotonic on $(0, \infty)$. The first proof of Theorem 1.1 is complete.

Second proof. To see the complete monotonicity of the function $h$, one writes

$h(t)-h(t+1)=e^{1 / t}-e^{1 /(t+1)}-\frac{1}{t^{2}}=\sum_{k=1}^{3} A_{k}(t)+\sum_{k=4}^{\infty} A_{k}(t)-\frac{1}{t^{2}}$

with

$A_{k}(t)=\frac{1}{k !} \frac{(t+1)^{k}-t^{k}}{t^{k}(t+1)^{k}}=\frac{1}{k !} \sum_{j=0}^{k-1} \frac{1}{(t+1)^{k} t^{k-j}}$.

Now since

$\sum_{k=1}^{3} A_{k}(t)-\frac{1}{t^{2}}=\frac{1}{6 t^{3}(t+1)^{3}}$

the difference $h(t)-h(t+1)$ is a sum of completely monotonic functions and hence completely monotonic on $(0, \infty)$. Finally, the function

$h(t)=\lim _{n \rightarrow \infty} \sum_{k=0}^{n}[h(t+k)-h(t+k+1)]$

is completely monotonic on $(0, \infty)$. The second proof of Theorem 1.1 is complete. 


\section{Proofs of Theorem 1.2}

It is general knowledge that the exponential function $e^{1 / z}$ for $z \in \mathbb{C}$ with $z \neq 0$ can be expanded into the Laurent series

$e^{1 / z}=\sum_{m=0}^{\infty} \frac{1}{m !} \frac{1}{z^{m}}, \quad z \neq 0$.

Therefore, it is clear that

$H_{k}(z)=\sum_{m=k+1}^{\infty} \frac{1}{m !} \frac{1}{z^{m}}, \quad z \neq 0$

and $x^{k+1} H_{k}(x)$ is completely monotonic on $(0, \infty)$. That is,

$\operatorname{deg}_{\mathrm{cm}}^{t}\left[H_{k}(t)\right] \geq k+1$.

Since, for any $\varepsilon>0$, the function

$x^{k+1+\varepsilon} H_{k}(x)=x^{\varepsilon} \sum_{m=0}^{\infty} \frac{1}{(m+k+1) !} \frac{1}{x^{m}}$

tends to $\infty$ as $x \rightarrow \infty$, we see that for any $\varepsilon>0$ the function $x^{k+1+\varepsilon} H_{k}(x)$ is not completely monotonic on $(0, \infty)$. That is,

$\operatorname{deg}_{\mathrm{cm}}^{t}\left[H_{k}(t)\right] \leq k+1$

Combining (3.3) and (3.4) leads to (1.5).

For $\Re z>0$ and $\Re k>0$, it was listed in [1, p. 255, 6.1.1] that

$\Gamma(z)=k^{z} \int_{0}^{\infty} t^{z-1} e^{-k t} \mathrm{~d} t$

This formula can be rearranged as

$\frac{1}{z^{w}}=\frac{1}{\Gamma(w)} \int_{0}^{\infty} t^{w-1} e^{-z t} \mathrm{~d} t$

for $\Re z>0$ and $\Re w>0$. Substituting the formula (3.5) into (3.2) yields

$H_{k}(z)=\int_{0}^{\infty}\left[\sum_{m=k+1}^{\infty} \frac{1}{m !} \frac{1}{\Gamma(m)} t^{m-1}\right] e^{-z t} \mathrm{~d} t=\frac{1}{k !(k+1) !} \int_{0}^{\infty}{ }_{1} F_{2}(1 ; k+1, k+2 ; t) t^{k} e^{-z t} \mathrm{~d} t$.

The integral representation (1.6) follows.

The function $H_{k}(z)$ can be rewritten as

$$
\begin{aligned}
H_{k}(z) & =\frac{1}{z^{k+1}} \sum_{m=0}^{\infty} \frac{1}{(k+1+m) !} \frac{1}{z^{m}} \\
& =\frac{1}{z^{k+1}}\left\{\frac{1}{(k+1) !}+\int_{0}^{\infty}\left[\sum_{m=1}^{\infty} \frac{1}{(k+1+m) !} \frac{1}{\Gamma(m)} t^{m-1}\right] e^{-z t} \mathrm{~d} t\right\} \\
& =\frac{1}{z^{k+1}}\left[\frac{1}{(k+1) !}+\int_{0}^{\infty} \frac{I_{k+2}(2 \sqrt{t})}{t^{(k+2) / 2}} e^{-z t} \mathrm{~d} t\right] .
\end{aligned}
$$

The integral representation (1.7) follows. Theorem 1.2 is thus proved. 


\section{Proof of Theorem $\mathbf{1 . 3}$}

When $k=0$, the integral representations (1.6) and (1.7) become

$e^{1 / z}=1+\int_{0}^{\infty} \frac{I_{1}(2 \sqrt{t})}{\sqrt{t}} e^{-z t} \mathrm{~d} t$

and

$e^{1 / z}=1+\frac{1}{z}\left[1+\int_{0}^{\infty} \frac{I_{2}(2 \sqrt{t})}{t} e^{-z t} \mathrm{~d} t\right]$

for $\Re z>0$. Hence, by (2.1) for $n=1$, the function $h(z)$ defined by (1.2) has the following integral representation

$h(z)=1+\int_{0}^{\infty}\left[\frac{I_{1}(2 \sqrt{u})}{\sqrt{u}}-\frac{u}{1-e^{-u}}\right] e^{-z u} \mathrm{~d} u$

and

$(-1)^{k} h^{(k)}(t)=\int_{0}^{\infty}\left[\frac{I_{1}(2 \sqrt{u})}{\sqrt{u}}-\frac{u}{1-e^{-u}}\right] u^{k} e^{-t u} \mathrm{~d} u$

for $k \geq 1$ are completely monotonic on $(0, \infty)$. The famous Hausdorff-Bernstein-Widder Theorem [11, p. 161, Theorem $12 \mathrm{~b}]$ states that a necessary and sufficient condition that $f(x)$ should be completely monotonic for $0<$ $x<\infty$ is that

$f(x)=\int_{0}^{\infty} e^{-x t} \mathrm{~d} \alpha(t)$,

where $\alpha(t)$ is non-decreasing and the integral converges for $0<x<\infty$. Consequently, the function in the bracket of (4.4) is not less than zero, that is,

$\frac{I_{1}(2 \sqrt{u})}{\sqrt{u}} \geq \frac{u}{1-e^{-u}}$

in which replacing $2 \sqrt{u}$ by $t$ yields the inequality (1.11). The proof of Theorem 1.3 is complete.

\section{Remarks}

In this final section, we would like to remark something.

Remark 5.1. Alternative proofs of Theorem 1.1 was given in [8, Theorem 3.1] and [10, Theorem 1.1].

Remark 5.2. The result (1.5) in Theorem 1.2 can be generalized as follows. Suppose that

$f(t)=\sum_{m=0}^{\infty} a_{m} t^{m}$

has infinite radius of convergence and that $a_{m} \geq 0$ for all $m$. Then

$\mathcal{H}_{k}(t)=f\left(\frac{1}{t}\right)-\sum_{m=0}^{k} \frac{a_{m}}{t^{m}}=\sum_{m=k+1}^{\infty} \frac{a_{m}}{t^{m}}$

is completely monotonic as well as

$t^{k+1} \mathcal{H}_{k}(t)=\sum_{m=k+1}^{\infty} \frac{a_{m}}{t^{m-k-1}}$,

so the function $\mathcal{H}_{k}(t)$ has the completely monotonic degree $k+1$ if $a_{k+1} \neq 0$ and, if $a_{k+1}=\cdots=a_{k+p}=0$ but $a_{k+p+1} \neq 0$, then $\mathcal{H}_{k}(t)$ has the completely monotonic degree $k+p+1$.

Remark 5.3. The integral representations (4.1) and (4.2) supply affirmative answers to an open problem posed in [13, p. 127, Section 4]. See also [12].

Remark 5.4. The inequality (1.11) in Theorem 1.3 or, equivalently, the inequality (4.6) has been further investigated in the subsequent papers $[7,8]$.

Remark 5.5. The formulas (1.6) and (4.1) have been applied in $[4,6]$ to combinatorics and number theory.

Remark 5.6. This paper is a corrected and extended version of the preprint [9]. 


\section{References}

[1] M. Abramowitz and I. A. Stegun (Eds), Handbook of Mathematical Functions with Formulas, Graphs, and Mathematical Tables, National Bureau of Standards, Applied Mathematics Series 55, 9th printing, Washington, 1970.

[2] B.-N. Guo and F. Qi, A completely monotonic function involving the tri-gamma function and with degree one, Appl. Math. Comput. 218 (2012), no. 19, 9890-9897; Available online at http://dx.doi.org/10.1016/j.amc.2012.03.075.

[3] B.-N. Guo and F. Qi, Refinements of lower bounds for polygamma functions, Proc. Amer. Math. Soc. 141 (2013), no. 3, 1007-1015; Available online at http://dx.doi.org/10.1090/S0002-9939-2012-11387-5.

[4] B.-N. Guo and F. Qi, Some integral representations and properties of Lah numbers, available online at http://arxiv.org/abs/1402.2367.

[5] S. Koumandos and H. L. Pedersen, Completely monotonic functions of positive order and asymptotic expansions of the logarithm of Barnes double gamma function and Euler's gamma function, J. Math. Anal. Appl. 355 (2009), no. 1, 33-40; Available online at http://dx.doi.org/10.1016/j.jmaa.2009.01.042.

[6] F. Qi, An explicit formula for Bell numbers in terms of Stirling numbers and hypergeometric functions, available online at http://arxiv.org/abs/1402.2361.

[7] F. Qi, Properties of modified Bessel functions and completely monotonic degrees of differences between exponential and trigamma functions, available online at http://arxiv.org/abs/1302.6731.

[8] F. Qi and C. Berg, Complete monotonicity of a difference between the exponential and trigamma functions and properties related to a modified Bessel function, Mediterr. J. Math. 10 (2013), no. 4, 1685-1696; Available online at http://dx.doi.org/10.1007/s00009-013-0272-2.

[9] F. Qi and S.-H. Wang, Complete monotonicity of a difference between the exponential and trigamma functions and completely monotonic degree of the exponential function, available online at http://arxiv.org/abs/1210.2012.

[10] F. Qi and X.-J. Zhang, Complete monotonicity of a difference between the exponential and trigamma functions, J. Korea Soc. Math. Educ. Ser. B Pure Appl. Math. 21 (2014), no. 2, 141-145; Available online at http://dx.doi.org/10.7468/jksmeb.2014.21.2.141.

[11] D. V. Widder, The Laplace Transform, Princeton University Press, Princeton, 1946.

[12] X.-J. Zhang, Integral Representations, Properties, and Applications of Three Classes of Functions, Thesis supervised by Professor Feng Qi and submitted for the Degree of Master of Science at Tianjin Polytechnic University in January 2013. (Chinese)

[13] X.-J. Zhang, F. Qi, and W.-H. Li, Properties of three functions relating to the exponential function and the existence of partitions of unity, Int. J. Open Probl. Comput. Sci. Math. 5 (2012), no. 3, 122-127. 